\title{
EDITORIAL
}

\section{A call for surgical case reports}

\author{
Ivo Brosens
}

Published online: 30 May 2009

(C) Springer-Verlag 2009

Many biomedical journals tend to frown upon case reports and, if published at all, often consider this section of the journal as a dustbin for papers of secondary importance. The Editorial Board of Gynecological Surgery, however, intends to promote and value the publication of case reports. Indeed, case reports on surgery are of major scientific value to the medical profession for a number of reasons. First, the publication of case reports is indispensable for and supplementary to evidence-based medicine, as it generates hypotheses that may be studied consecutively in higher level of evidence designs. Secondly, the vast majority of reports are written by junior doctors, often attempting for the first time in their career to distil the scientific or medical significance of their observations and to share these with their peers. When the work is of outstanding quality and conform to strict criteria, such reports deserve to be published in the foremost international journals. Thirdly, case reports contribute to innovation and expansion of surgical knowledge. This Journal has longstanding tradition of publishing case reports and it is a pleasant surprise to find that, in a continuous series of 55 case reports in Gynecological Surgery, six (11\%) claimed to be the "first ever" report. These publications originated

\section{Brosens $(\triangle)$}

LIFE, Leuven Institute for Fertility and Embryology,

Tiensevest 168,

3000 Leuven, Belgium

e-mail: ivo.brosens@med.kuleuven.ac.be from UK, Turkey, and Denmark and described either a new surgical approach or hitherto unrecognized surgical events or complications. Fourthly, $60 \%$ of case reports were illustrated, which demonstrate their potential value for medical education. Fifthly, the scientific value of case reports is further illustrated by the fact this section, corrected for the number of pages, is cited as frequently as the review section or the section with original articles. Finally, case reports remain an invaluable source of information for the management of cases with unexpected or unusual presentation or rare surgical complications.

It is therefore our intention to restore Case Reports as a major section in this Journal for which manuscripts will be selected on the basis of originality and scientific interest. The format of case reports is similar to that of any other original article but limited to 2,000 words, and should include an Abstract (maximum of 100 words) with keywords, Introduction, Case presentation, Discussion, Conclusion, and a list of no more than ten references. The number of illustrations is limited to three.

As the selection of case reports is based on strict criteria of major interest, we offer the author(s) of a case report a fast tract review process, which will take not more than a week to receive the information on the Editor's decision.

There is no doubt that the publication of high-quality case reports contributes to the expansion of our knowledge and expertise in gynecological surgery, which has always been the primary objective of this journal. 